\title{
Acknowledgments
}

\section{Algorithmic Decision Theory}

\section{Raymond Bisdorff}

Computer Science and Communications RU Faculty of Sciences, Technology \& Communication University of Luxembourg

http://charles-sanders-peirce.uni.lu/bisdorff/

ICOCBA'2012, Kolkata December 2012
This presentation contains ideas that are not only the author's.

They have been borrowed from friends and colleagues :

Denis Bouyssou, Luis Dias,

Claude Lamboray, Patrick Meyer,

Vincent Mousseau, Alex Olteanu,

Marc Pirlot, Thomas Veneziano,

and especially,

Alexis Tsoukiàs.

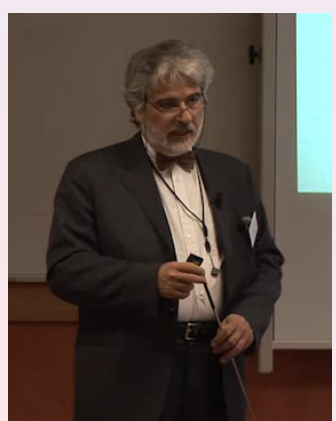

A. Tsoukiàs

Their help is gratefully acknowledged.

\section{Content}

Algorithmic Decision Theory

1. The GDRI AlgoDeC

History

Members

Online Resources

2. Introduction to Algorithmic Decision Theory

Formulating decision problems

Identifying a decision problematique

Selecting the evaluation model

3. Constructing decision recommendations

Selecting and ranking

K-sorting

Clustering
- From 2007 to 2011 the COST Action IC0602 Algorithmic Decision Theory (coordinated by Alexis Tsoukiàs) put together researchers coming from different fields such as Decision Theory, Discrete Mathematics, Theoretical Computer Science and Artificial Intelligence in order to improve decision support in the presence of massive data bases, combinatorial structures, partial and/or uncertain information and distributed, possibly interoperating decision makers.

- Working Groups :

- Uncertainty and Robustness in Planning and Dcision Making

- Decision Theoretic Artificial Intelligence

- Preferences in Reasoning and Decision

- Knowledge extraction and Learning 


\section{The GDRI AlgoDeC}

- The Groupement de Recherche International GDRI ALGODEC follows from the COST Action IC0602 Algorithmic Decision Theory and federates a number of research institutions strongly interested in this research aera.

- The aim is networking the many initiatives undertaken within this domain, organising seminars, workshops and conferences, promoting exchanges of people (mainly early stage researchers), building up an international community in this exciting research area.

- http://www.gdri-algodec.org/

\section{AlgoDec Members}

The GDRI ALGoDEC was established in 2011 around the following institutions :

DIMACS - Rutgers University (Rutgers, USA)

CNRS - Centre National de la Recherche Scientifique (FR)

LAMSADE - Université Paris-Dauphine (Paris, FR)

LIP6 - Université Pierre et Marie Curie (Paris, FR)

CRIL - Université d'Artois (Lens, FR)

FNRS - Fonds National de la Recherche Scientifique (BE)

MATHRO - Université de Mons (BE)

SMG - Université Libre de Bruxelles (BE)

FNR - Fonds National de la Recherche (LU)

ILIAS - University of Luxembourg (LU)

DEIO - Universidad Rey Juan Carlos (Madrid, ES)

GDRI AlgoDec

$\stackrel{\infty}{\circ}, \circ$

\section{AlgoDec Coordination}

The activities of the GDRI ALGODEC are steered by the following Committee :

\section{Dr. Denis BOUYSSOU, Coordinator, LAMSADE (Paris)}

Prof. Raymond BISDORFF, ILIAS (Luxembourg)

Prof. Yves DE SMET, SMG (Bruxelles)

Prof. Pierre MARQUIS, CRIL (Lens, FR)

Prof. Patrice PERNY, LIP6 (Paris)

Prof. Marc PIRLOT, MATHRO (Mons, BE)

Prof. David RIOS INSUA, DEIO (Madrid)

Prof. Fred ROBERTS, DIMACS (Rutgers USA)

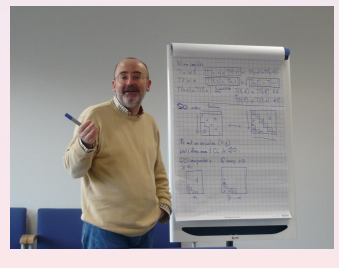

D. Bouyssou
GDRI AlgoDec

$\circ \circ$

\section{AlgoDec Activities}

- EURO working group on Multiple Criteria Decision Aid

- The 1st and 2nd International Conference on Algorithmic Decision Theory

- The Decision Deck project

- EURO working group on Preference Handling

- The DIMACS Special Focus on Algorithmic Decision Theory

- The 4th International Workshop on Computational Social Choice

- Smart Cities Workshop

- Workshop on Policy Analytics

- DA2PL'2012 workshop on Multiple Criteria Decision Aid and Preference Learning 


\section{GDRI AlgoDeC Online Resources}

Decision Deck Online Resources

Tutorials and course materials on http://www.algodec.org.

44 contributions on Algorithmic Decision Theory contain videos and presentation materials originating from the tutorials and courses who took place at the meetings and doctoral schools organised by the COST Action IC0602 Algorithmic Decision Theory.

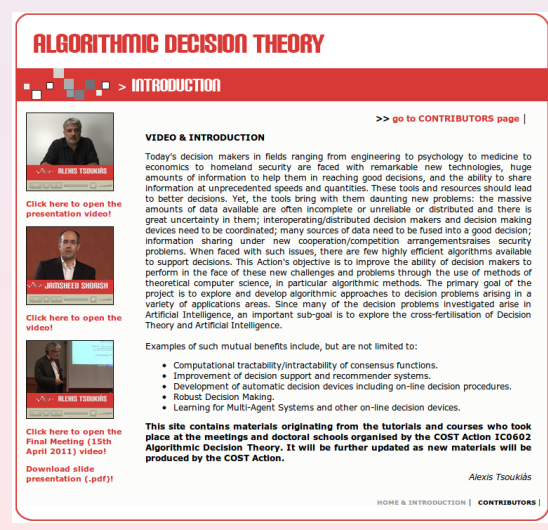

XMCDA data encoding resources and webservices on http://www.decision-deck.org.

The DeCision DeCK project aims at collaboratively developing Open Source software tools implementing MultiCriteria Decision Aid (MCDA) techniques which are meant to support complex decision aid processes and are interoperable in order to create a coherent ecosystem.

1. The GDRI ALGODEC

History

Members

Online Resources

2. Introduction to Algorithmic Decision Theory

Formulating decision problems

Identifying a decision problematique

Selecting the evaluation model

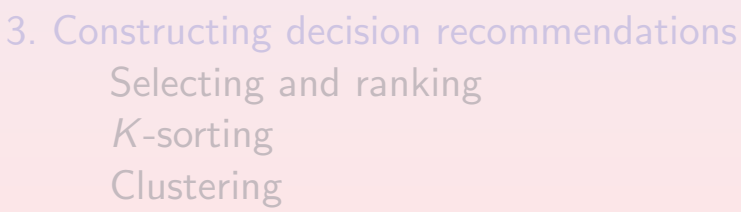

\section{Characterizing Decision Problems}

A decision problem will be a tuple $\mathcal{P}=(D, A, O, F, \Omega)$ where

1. $D$ is a group of $d=1, \ldots$ decision makers :

2. $A$ is a set of $n=2, \ldots$ decision actions or alternatives;

3. $O$ is a set of $O=1, \ldots$ decision objectives;

4. $F$ is a set of $m=1, \ldots$ attributes or performance criteria (to be maximised or minimised) with respect to decision objective obj $\in O$;

5. $\Omega$ is a set of $\omega=1, \ldots$ potential states of the world or context scenarios. 


\section{Types of Decision Problems}

We may distinguish different types of decision problems along three directions :

- Single or multiple objectives/criteria,

- Single or multiple decision makers,

- Single or multiple context scenarios.

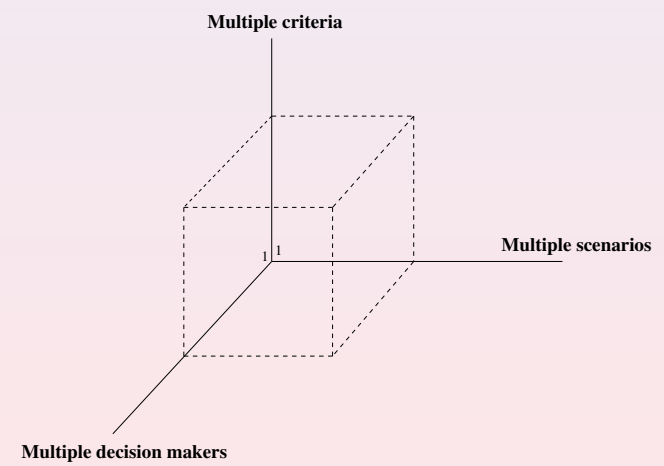

Multiple decision makers

Formulating the Decision Alternatives

- Small set of individual decision alternatives;

- Large set of alternatives consisting in the combination of given features;

- Infinite set of decision alternatives;

- Portfolios of potential alternatives;

- Stream of potential decision alternatives;

- Critical decision alternatives (emergency or disaster recovering).

\section{Decision aiding approach}

\begin{tabular}{c|c|c|c|c|c}
\hline $\begin{array}{c}\text { Situating } \\
\text { the problem }\end{array}$ & \multicolumn{2}{|c|}{$\begin{array}{c}\text { Formulating } \\
\text { the problem }\end{array}$} & \multicolumn{2}{|c|}{$\begin{array}{c}\text { Selecting the } \\
\text { evaluation model }\end{array}$} & $\begin{array}{c}\text { Constructing } \\
\text { the final } \\
\text { recommendation }\end{array}$ \\
\hline & $\begin{array}{c}\text { Decision } \\
\text { Objects }\end{array}$ & $\begin{array}{c}\text { Decision } \\
\text { Problem }\end{array}$ & The model & $\begin{array}{c}\text { Tunning } \\
\text { the model }\end{array}$ & \\
\cline { 2 - 4 } Actors & Alternatives & Ranking & $\begin{array}{c}\text { Value } \\
\text { Functions }\end{array}$ & directly & \\
Stakes & Corting & $\begin{array}{c}\text { Outranking } \\
\text { Relations }\end{array}$ & indirectly & \\
Resources & & & & Method \\
\hline [Tsoukias:2007] & & & &
\end{tabular}

\section{Formulating decision objectives and criteria}

- Identifying the strategic objectives of the decision making problem

- Identifying all objective consequences of the potential decision actions, measured on :

- Discrete ordinal scales?

- Numerical, discrete or continuous scales?

- Interval or ratio scales?

- Each consequence is associated with a strategic objective

- to be minimized (Costs, environmental impact, energy consumption, etc);

- to be maximised (Benefits, energy savings, security and reliability, etc). 


\section{Decision Problematiques}

From an algorithmic point of view, we may distinguish the following types of decision problems :

- Choice : selecting the $k$ best (or worst) choices, $k=1, \ldots$;

- Ranking : Linearly ordering $k=1, \ldots, n$ choices from the best to the worst ;

- Sorting or Rating : Supervised clustering into $k=2, \ldots$ predefined, and usually linearly ordered, sort categories;

- Clustering : unsupervised grouping into an unknown number $k=2, \ldots$ of clusters.

\section{Epistemic truth semantics of the $r$-valuation}

Let $x \succsim y$ and $x^{\prime} \succsim y^{\prime}$ be two preferential assertions :

$r(x \succsim y)=+1$ means that assertion $x \succsim y$ is certainly valid,

$r(x \succsim y)=-1$ means that assertion $x \succsim y$ is certainly invalid,

$r(x \succsim y)>0$ means that assertion $x \succsim y$ is more valid than invalid,

$r(x \succsim y)<0$ means that assertion $x \succsim y$ is more invalid than valid,

$r(x \succsim y)=0$ means that

validity of assertion $x \succsim y$ is indeterminate,

$r(x \succsim y)>r\left(x^{\prime} \succsim y^{\prime}\right)$ means that

assertion $x \succsim y$ is more valid than assertion $x^{\prime} \succsim y^{\prime}$,

$r(\neg x \succsim y)=-r(x \succsim y)$

logical (strong) negation by changing sign,

$r\left(x \succsim y \vee x^{\prime} \succsim y^{\prime}\right)=\max \left(r(x \succsim y), r\left(x^{\prime} \succsim y^{\prime}\right)\right)$

logical disjonction via the max operator,

$r\left(x \succsim y \wedge x^{\prime} \succsim y^{\prime}\right)=\min \left(r(x \succsim y), r\left(x^{\prime} \succsim y^{\prime}\right)\right)$

logical conjonction via the min operator.

\section{$X$ : Finite set of $n$ alternatives}

$x \succsim y$ : Alternative $x$ outranks alternative $y$ if

1. there is a (weighted) majority of voters or criteria supporting that $x$ is at least as good as $y$, and

2. no veto or considerable negative performance difference between $x$ and $y$ is observed on a discordant criterion.

$x \nsucceq y$ : Alternative $x$ does not outrank alternative $y$ if

1. there is a (weighted) majority of voters or criteria supporting that $x$ is not at least as good as $y$, and

2. no counter-veto or considerable positive performance difference between $x$ and $y$ is observed on a discordant crterion.

$r(x \succsim y)$ represents a bipolar, i.e. concordance versus discordance, valuation in $[-1,1]$ characterizing the epistemic truth of affirmative assertion $x \succsim y$.

$17 / 40$

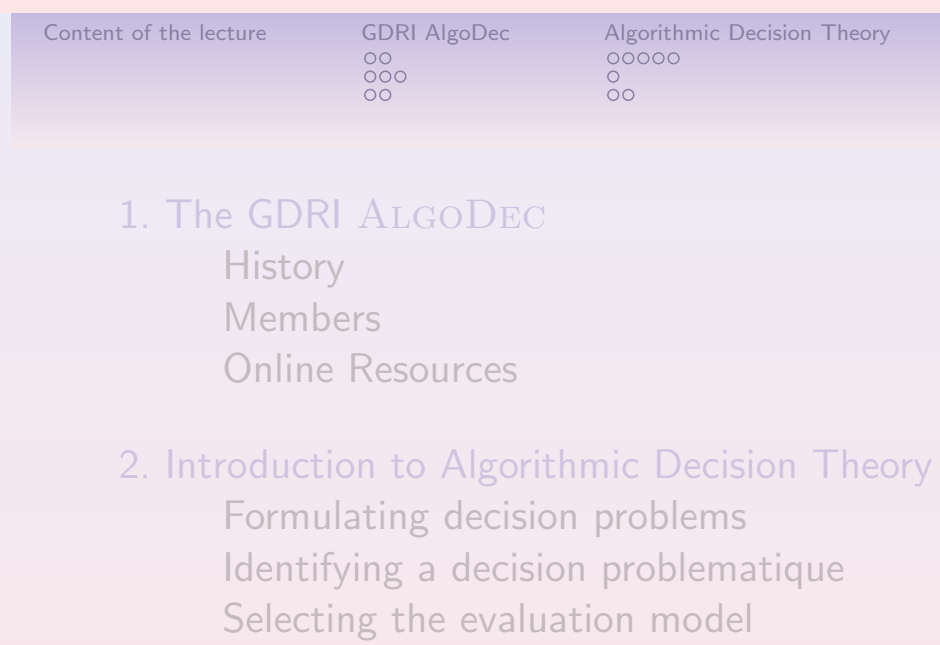

3. Constructing decision recommendations

Selecting and ranking

K-sorting

Clustering 


\section{Useful choice qualifications}

- A choice problem traditionally consists in the search for a single best alternative ;

- Pragmatic Best Choice Recommendation - BCR - principles :

$P_{1}$ : Non retainement for well motivated reasons;

$P_{2}$ : Recommendation of minimal size;

$P_{3}$ : Stable (irreducible) recommendation;

$P_{4}$ : Effectively best choice;

$P_{5}$ : Recommendation maximally supported by the given preferential information.

- The decision aiding process progressively uncovers the best single choice via more and more refined choice recommendations;

- The process stops when the decision maker is ready to make her final decision.
Let $Y$ be a non-empty subset of $X$, called a choice.

- $Y$ is said to be outranking (resp. outranked) iff $x \notin X \Rightarrow \exists y \in Y: r(x \succsim y)>0)$

- $Y$ is said to be independent iff for all $x \neq y$ in $Y$ we have $X$ $r(x \succsim y) \leqslant 0)$.

- $Y$ is called an outranking kernel (resp. outranked kernel) iff it is an outranking (resp. outranked) and indendent choice.

- $Y$ is called an outranking hyperkernel (resp. outranked hyperkernel) iff it is an outranking (resp. outranked) choice which consists of independent chordless circuits of odd length $\geqslant 1$

References : Roy \& Bouyssou (1993), Bisdorff, Roubens \& Meyer (2008).

\section{Translating BCR principles into choice qualifications}

$P_{1}:$ Non-retainment for well motivated reasons.

A BCR is an outranking choice.

$P_{2+3}$ : Minimal size \& stable.

A BCR is a hyperkernel.

$P_{4}$ : Effectivity.

A BCR is a stricly more outranking than outranked choice.

$P_{5}$ : Maximal credibility.

A BCR has maximal determinateness.

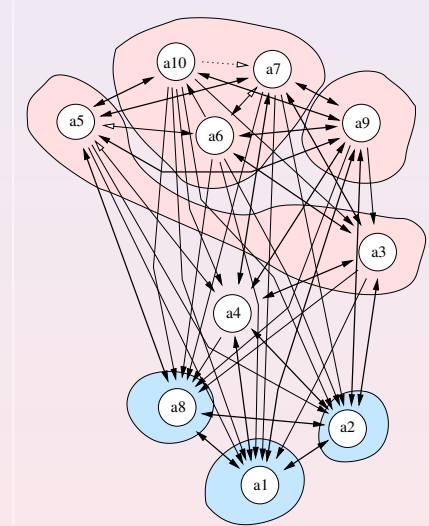

Theorem (BCR Decisiveness, Bisdorff et al. 2008)

Any bipolar strict outranking digraph contains at least one outranking and one outranked hyperkernel.

\section{The Linear Ranking Problematique}

- A ranking problem traditionally consists in the search for a linear ordering of the set of alternatives ;

- A particular ranking is computed with the help of a ranking rule which aggregates preferences over all decision makers and/or criteria into a global (weak) order based, either on (rank) scoring (Borda), or, on (pairwise) voting procedures (Kemeny, Slater, Copeland, Kohler, Ranked Pairs);

- Characteristic properties of ranking rules :

1. A ranking rule is called Condorcet-consistent when the following holds: If the majority relation is a linear order, then this linear order is the unique solution of the ranking rule;

2. A ranking rule is called B-ordinal if its result only depends on the order of the majority margins $B$;

3. A ranking rule is called M-invariant if its result only depends on the majority relation $M$

Reference : Cl. Lamboray $(2007,2009,2010)$ 


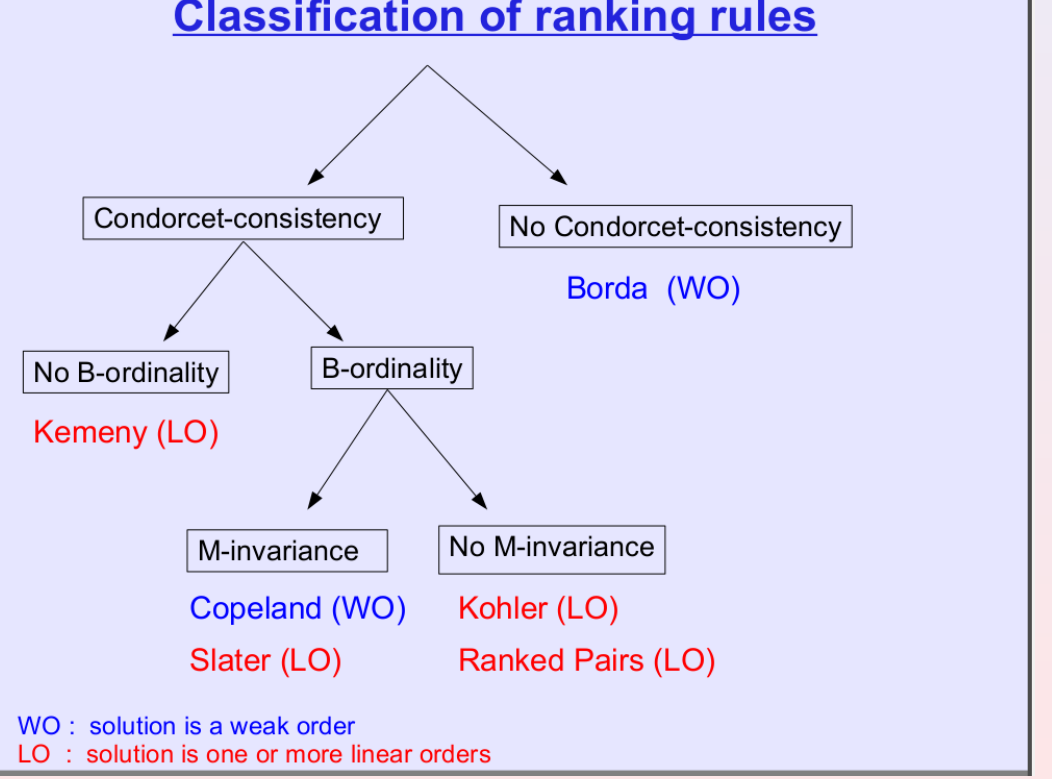

- A sorting problem consists in a supervised partitioning of the set of alternatives into $k=2, \ldots$ ordred sorts or categories.

- Usually, a sorting procedure is designed to deal with an absolute evaluation model, whereas choice and ranking algorithms essentially rely on relative evaluation models.

- A crucial problem, hence, lies in the definition of the given categories, i.e., of the evaluation norms that define each sort category.

- Two type of such norms are usually provided :

- Delimiting (min-max) evaluation profiles;

- Central representatives.

\section{Sorting with delimiting norms}

Sort category $K$ is delimited by an interval $\left[m^{k} ; M^{k}[\right.$ on a performance measurement scale; $x$ is a measured performance. We may distinguish three sorting situations :

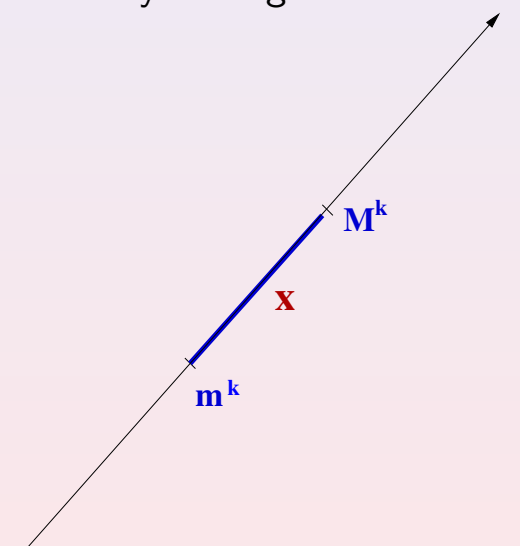

1. $x<m^{k}$ (and $x<M^{k}$ ) The performance $x$ is lower than category $K$;

2. $x \geqslant m^{k}$ and $x<M^{k}$ The performance $x$ belongs to category $K$;

3. $\left(x \geqslant m^{k}\right.$ and $) x \geqslant M^{k}$ The performance $x$ is higher than category $K$.

If the relation $<$ is the dual of $\geqslant$, it will be sufficient to check that $x \geqslant m_{k}$ as well as $x \ngtr M_{k}$ are true for $x$ to be a member of $K$.
Decision aiding 0000000

\section{Notations}

- $X=\{x, y, z, \ldots\}$ is a finite set of objects to be sorted.

- $F=\{1, \ldots, n\}$ is a finite and coherent family of performance criteria.

- For each criterion $i$ in $F$, the objects are evaluated on a real performance scale $\left[0 ; M_{i}\right]$,

supporting an indifference threshold $q_{i}$

and a preference threshold $p_{i}$ such that $0 \leqslant q_{i}<p_{i} \leqslant M_{i}$.

- The performance of object $x$ on criterion $i$ is denoted $x_{i}$.

- Each criterion $i$ in $F$ carries a rational significance $w_{i}$ such that $0<w_{i}<1.0$ and $\sum_{i \in F} w_{i}=1.0$. 
Each criterion $i$ is characterising a double threshold order $\geqslant_{i}$ on $X$ in the following way :

$$
r(x \geqslant i y)=\left\{\begin{array}{l}
+1 \quad \text { if } \quad x_{i}+q_{i} \geqslant y_{i} \\
-1 \quad \text { if } \quad x_{i}+p_{i} \leqslant y_{i} \\
0 \text { otherwise. }
\end{array}\right.
$$

+1 signifies $x$ is performing at least as good as $y$ on criterion $i$,

-1 signifies that $x$ is not performing at least as good as $y$ on criterion $i$.

0 signifies that it is unclear whether, on criterion $i, x$ is performing at least as good as $y$.
Each criterion $i$ contributes the significance $w_{i}$ of his "at least as good as" characterisation $r\left(\geqslant_{i}\right)$ to the global characterisation $r(\geqslant)$ in the following way :

$$
r(x \succsim y)=\sum_{i \in F}\left[w_{i} \cdot r(x \geqslant i y)\right]
$$

$r>0$ signifies $x$ is globally performing at least as good as $y$,

$r<0$ signifies that $x$ is not globally performing at least as good as $y$,

$r=0$ signifies that it is unclear whether $x$ is globally performing at least as good as $y$.

\section{Performing marginally and globally less than}

Each criterion $i$ is characterising a double threshold order $<_{i}$ (less than) on $X$ in the following way :

$$
r\left(x<_{i} y\right)=\left\{\begin{array}{l}
+1 \quad \text { if } x_{i}+p_{i} \leqslant y_{i} \\
-1 \text { if } x_{i}+q_{i} \geqslant y_{i} \\
0 \text { otherwise. }
\end{array}\right.
$$

And, the global less than relation $(\prec)$ is defined as follows :

$$
r(x \prec y)=\sum_{i \in F}\left[w_{i} \cdot r\left(x<_{i} y\right)\right]
$$

Proposition (Bisdorff 2012)

The global "less than" relation $\prec$ is the dual $(\mathcal{Z})$ of the global "at least as good as" relation $\succsim$.

\section{Characterising the category $K$ membership}

Let $m^{k}=\left(m_{1}^{k}, m_{2}^{k}, \ldots, m_{p}^{k}\right)$ denote the lower limits and $M^{k}=\left(M_{1}^{k}, M_{2}^{k}, \ldots, M_{p}^{k}\right)$ the corresponding upper limits of category $K$ on the criteria.

\section{Proposition}

That object $x$ belongs to category $K$ may be characterised as follows:

$$
r(x \in K)=\min \left(r\left(x \succsim m^{k}\right),-r\left(x \succsim M^{k}\right)\right)
$$


- Clustering is an unsupervised learning method that groups a set of objects into clusters.

- Properties :

- Unknown number of clusters;

- Unknown characteristics of clusters ;

- Only the relations between objects are used;

- no relation to external categories are used.

- Usually used in exploratory analysis and for cognitive artifacts.

Clustering decision aid

\begin{tabular}{|c|c|c|c|c|c|}
\hline $\begin{array}{l}\text { Situating } \\
\text { the problem }\end{array}$ & \multicolumn{2}{|c|}{$\begin{array}{l}\text { Formulating } \\
\text { the problem }\end{array}$} & \multicolumn{2}{|c|}{$\begin{array}{l}\text { Selecting the } \\
\text { evaluation model }\end{array}$} & $\begin{array}{c}\text { Constructing } \\
\text { the final }\end{array}$ \\
\hline \multirow[b]{2}{*}{ Actors } & $\begin{array}{l}\text { Decision } \\
\text { Objects }\end{array}$ & $\begin{array}{l}\text { Decision } \\
\text { Problem }\end{array}$ & The model & $\begin{array}{l}\text { Tunning } \\
\text { the model }\end{array}$ & \multirow{4}{*}{ Method } \\
\hline & Alternatives & $\begin{array}{l}\text { Choice } \\
\text { Ranking }\end{array}$ & $\begin{array}{l}\text { Value } \\
\text { Functions }\end{array}$ & directly & \\
\hline Stakes & & Sorting & & & \\
\hline Resources & Criteria & $\begin{array}{l}\text { Clustering } \\
\text { classical } \\
\text { relation } \\
\text { ordered }\end{array}$ & $\begin{array}{c}\text { Outranking } \\
\text { Relations }\end{array}$ & indirectly & \\
\hline
\end{tabular}

[Tsoukias:2007] 


\section{Bibliography I}

\section{CLIP (CLustering using Indifferences and Preferences)}

1. Grouping on indifferences (internal);

- finding an initial partition;

- high concentration of indifference relations inside clusters;

- low concentration of indifference relations between clusters;

- graph theoretic inspired method using cluster cores;

2. Refining on preferences (external);

- searching for the optimal result;

- strengthen relations between clusters;

- meta-heuristic approach.

P. Perny and A. Tsoukiàs, Decision analysis and artificial intelligence. European Journal of Operational Research, vol. 160(3), 300 pages, 2005.

A. Tsoukiàs, From Decision Theory to Decision Aiding Methodology. European Journal of Operational Research, vol. 187 (2008) 138 - 161

R. Bisdorff, On polarizing outranking relations with large performance differences. Journal of Multi-Criteria Decision Analysis DOI : $10.1002 /$ mcda. $14721-20$

R. Bisdorff, P. Meyer and M. Roubens, RUBIS : a bipolar-valued outranking method for the choice problem. 4OR, A Quarterly Journal of Operations Research, Springer-Verlag, Volume 6 Number 2 (2008) 143-165.

R. Bisdorff, Concordant Outranking with multiple criteria of ordinal significance. 4OR, A Quarterly Journal of Operations Research, Springer-Verlag, Volume 2 Number 4 (2004) 293-308.
F. Rossi and A. Tsoukiàs, Algorithmic Decision Theory. Proceedings of the 1st International Conference ADT2009, LNAI 5783, Springer Verlag, Berlin, 460 pages, 2009.

D. Bouyssou, Th. Marchant, M. Pirlot, A. Tsoukiàs and Ph. Vincke, Evaluation and Decision Models : stepping stones for the analyst. Springer Verlag, Berlin, 2006.

D. Bouyssou, Th. Marchant, P. Perny, M. Pirlot, A. Tsoukiàs and Ph. Vincke, Evaluation and Decision Models : a critical perspective. Kluwer Academic, Dordrecht, 2000.

D.S. Roberts and A. Tsoukiàs, Special Issue on Voting and Preference Modelling. Mathematical Social Sciences, vol. 57, 118 pages, 2009.

F.S. Roberts and A. Tsoukiàs, Special Issue on Computer Science and Decision Theory. Annals of Operations Research, vol. 163, 270 pages, 2008.

\section{Bibliography III}

L.C. Dias and C. Lamboray, Extensions of the prudence principle to exploit a valued outranking relation. European Journal of Operational Research Volume 201 Number 3 (2010) 828-837.

C. Lamboray, A prudent characterization of the Ranked Pairs Rule. Social Choice and Welfare 32 (2009) 129-155.

㐭 C. Lamboray, A comparison between the prudent order and the ranking obtained with Borda's, Copeland's, Slater's and Kemeny's rules. Mathematical Social Sciences 54 (2007) 1-16.

R. Bisdorff, P. Meyer and A. Olteanu, A Clustering Approach using Weighted Similarity Majority Margins. Tang et al. (Eds.) : Advanced Data Mining and Applications ADMA 2011, Part I, Springer-Verlag LNAI 7120 (2011) 15-28. 